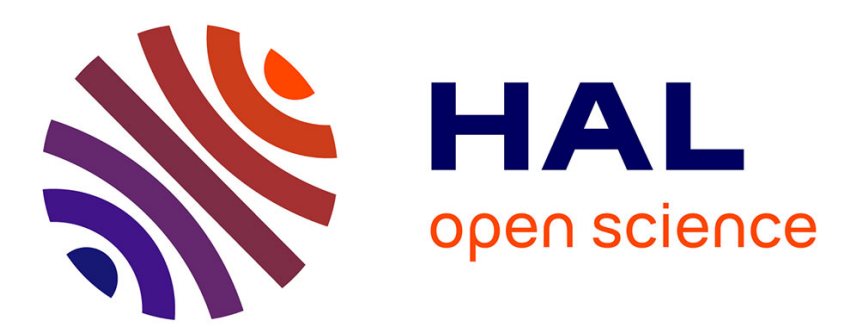

\title{
Reducing localisation overhead: a ranging protocol and an enhanced algorithm for UWB-based WSNs
}

Réjane Dalce, Adrien van den Bossche, Thierry Val

\section{To cite this version:}

Réjane Dalce, Adrien van den Bossche, Thierry Val. Reducing localisation overhead: a ranging protocol and an enhanced algorithm for UWB-based WSNs. 81st IEEE Vehicular Technology Conference (VTC 2015 - Spring), May 2015, Glasgow, United Kingdom. 10.1109/VTCSpring.2015.7146048. hal-01387818

\section{HAL Id: hal-01387818 \\ https://hal.science/hal-01387818}

Submitted on 26 Oct 2016

HAL is a multi-disciplinary open access archive for the deposit and dissemination of scientific research documents, whether they are published or not. The documents may come from teaching and research institutions in France or abroad, or from public or private research centers.
L'archive ouverte pluridisciplinaire HAL, est destinée au dépôt et à la diffusion de documents scientifiques de niveau recherche, publiés ou non, émanant des établissements d'enseignement et de recherche français ou étrangers, des laboratoires publics ou privés. 


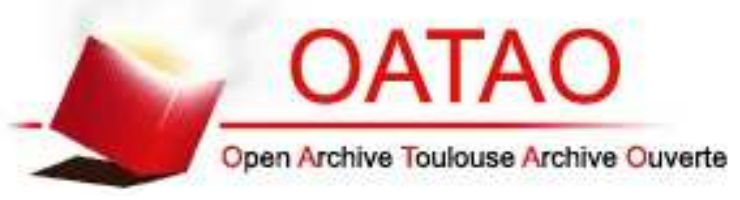

\section{Open Archive TOULOUSE Archive Ouverte (OATAO)}

OATAO is an open access repository that collects the work of Toulouse researchers and makes it freely available over the web where possible.

This is an author-deposited version published in : http://oatao.univ-toulouse.fr/ Eprints ID : 15218

The contribution was presented at VTC 2015 - Spring: http://www.ieeevtc.org/vtc2015spring/

To cite this version : Dalce, Rejane and Van den Bossche, Adrien and Val, Thierry Reducing localisation overhead: a ranging protocol and an enhanced algorithm for UWB-based WSNs. (2015) In: 81 st IEEE Vehicular Technology Conference (VTC 2015 - Spring), 11 May 2015 - 14 May 2015 (Glasgow, Scotland, United Kingdom).

Any correspondence concerning this service should be sent to the repository administrator: staff-oatao@listes-diff.inp-toulouse.fr 


\title{
Reducing localisation overhead: a ranging protocol and an enhanced algorithm for UWB-based WSNs
}

\author{
Rejane Dalce, Adrien van den Bossche, Thierry Val \\ Institut de Recherche en Informatique de Toulouse \\ UMR 5505 - CNRS, Université de Toulouse \\ Toulouse, France \\ Email: $\{$ dalce,vandenbo,val $\} @$ irit.fr
}

\begin{abstract}
The ability for the nodes in a Wireless Sensor Network to determine their position is a desirable trait. Routing as well as other client applications can benefit from this information. In this paper, we introduce the results obtained from our UWB-based prototype. We implemented two adaptations of the Symmetric Double-Sided Two-Way Ranging (SDS-TWR) protocol, namely Sequential Symmetric Double-Sided Two-Way Ranging (SSDS-TWR), and Parallel Double-Sided Two-Way Ranging (PDS-TWR), the latter being one of our contributions. PDS-TWR significantly reduces the overhead associated with ranging. We also introduce the enhanced version of our localisation algorithm, inter-Ring Localisation Algorithm (iRingLA), which is a good alternative for conventional trilateration. This new version improves the ability to compute the position when thin rings are used by focusing on the exact intersection: the number of test points remains small and the algorithm can be implemented on computationally constrained platforms. Using PDS-TWR and 2 anchors, we obtained a 2D localisation error of $79 \mathrm{~cm}$ in an indoor environment.
\end{abstract}

\section{INTRODUCTION}

As we are moving towards the Internet of Things, the ability to map the collected data to a specific location becomes essential. In addition to transporting information, the network must provide a localisation service. Other applications and clients will then use this service's output to enhance performance, offer innovative interaction and other services. Our research therefore focuses on enabling localisation capabilities in nodes of a Wireless Sensor Network (WSN).

This localisation service can be broken down in two: ranging and position estimation. Ranging involves a protocol which will enable the nodes in the network to collect relevant information. We focus on Time Of Flight (TOF) as this information is far more reliable than Received Signal Strength (RSS). Three protocols can be considered as well-known in this domain: Time Of Arrival, Two-Way Ranging and Symmetric Double-Sided Two-Way Ranging (SDS-TWR).

The apparently lightest ranging protocol is TOA (figure 1(a)). It involves a mobile and a reference node, also called anchor. The mobile sends a message to the anchor and marks the emission time. The anchor measures the reception time and in another message, informs the mobile. This node can then compute the TOF using equation 1.

$$
T O F=t_{2}-t_{1}
$$

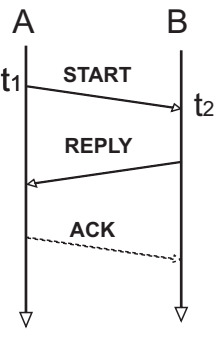

(a) Time Of Arrival

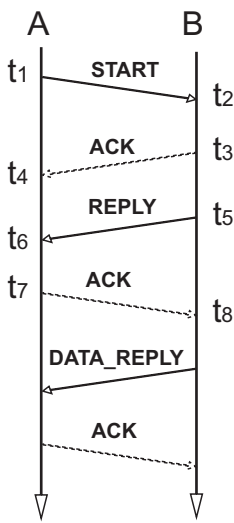

(c) Symmetric Double-Sided Two-Way Ranging (CSS)

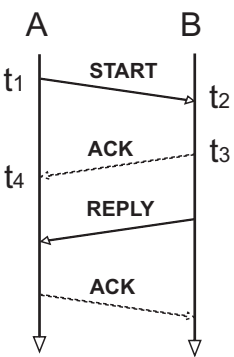

(b) Two-Way Ranging

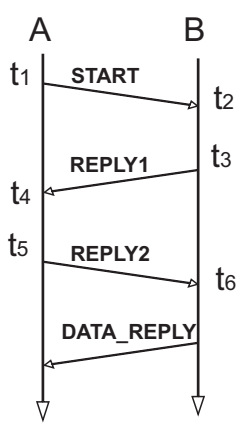

(d) Symmetric Double-Sided Two-Way Ranging (UWB)

Fig. 1. Ranging protocols

This ranging protocol seems very simple but it relies on finegrained synchronisation between the anchor and mobile. TwoWay Ranging protocol has been proposed in order to avoid solving the synchronisation problem (figure 1(b)). Instead of combining timestamps, the mobile uses the durations measured from each side, using a data frame and its ACK frame. The anchor's data is sent later to the mobile through another message.

Another protocol known as Symmetric Double-Sided TwoWay Ranging (SDS-TWR) [1] has later been proposed (figure 1(c)). Using two TWRs, it reduces the impact of clock skew on the ranging results. Depending on the available hardware platform, the number of messages may change. For example, on a Chirp Spread Spectrum (CSS) platform [2], due to 
timestamping limitations, only the version of figure 1(c) can be implemented. The TOF can be computed with equation 2 .

$$
t_{\text {flight }}=\frac{t_{4}-t_{1}-\left(t_{3}-t_{2}\right)+\left(t_{8}-t_{5}\right)-\left(t_{7}-t_{6}\right)}{4}
$$

On our Ultra-Wide Band (UWB) platform [3], ranging can still be performed without the ACK frames.

Many adaptations of SDS-TWR have been proposed, the most natural evolution being the sequential execution of the protocol with various anchors, which we will call Sequential SDS-TWR (SSDS-TWR) [9].

The work in [4] introduces the Burst protocol where $\mathrm{k}$ executions of SDS-TWR take place between a mobile and an anchor but are interweaved: the mobile sends the Start frame $k$ times, the anchor replies $k$ times and so on. The simulations and mathematical model confirm that this protocol, reduces the localisation error but increases the medium usage of the localisation service.

Double Two-Way Ranging (D-TWR) protocol is proposed in [5]. The mobile emits two consecutive Start messages and the anchor only replies once. The objective is to reduce overhead and error and enhance processing speed.

In [6], the authors present SDS-TWR-Multiple Acknowledgement. In this case, the anchor sends multiple ACK frames to the single Start message of the mobile. The idea is to reduce ranging time while collecting many samples related to the same anchor. In this solution, the anchor computes the TOF and transmits it to a localisation server. This protocol has been compared to classic SDS-TWR and TWR in [7] in terms of robustness to clock drift and duration of the ranging phase.

The work in [8] introduces AVSR which tries to share the Start message between $n$ anchors. The mobile broadcasts this frame and announces the existence of s slots. Each anchor will randomly choose a slot and transmit its ACK frame. Before this transmission can take place, the anchors sense the medium to detect the preamble. If another node is currently transmitting, the anchor will choose one of the remaining slots or cancel its participation should there be no more slots. This work is interesting as it considers the presence of multiple mobiles: an acquisition slot is defined where all mobiles send their Start message.

Most of these propositions share a common objective which is to produce the most precise and accurate distance estimation. In this article, we will study the problem from a network point of view. The remainder of this paper is organised as follows: section II introduces the problem at hand, then section III describes our proposed protocol. Section IV compares our protocol to the existing solutions using analytical models and section $\mathrm{V}$ discusses prototype results obtained using our protocol. Section VI then concludes this paper.

\section{Problem StATEMENT}

A WSN node is usually limited in terms of processing power and energy. Although some nodes may be fixed, most of them are mobile and must therefore use their battery widely. The ranging protocol requires the nodes to receive and transmit messages. These two actions have a significant impact on the global power consumption of the node, radio reception having the highest cost. This protocol must provide mechanisms to keep the induced power consumtion to a minimum.

Another important aspect is overhead. The main objective when deploying a WSN is collecting data and interacting with the environment. The traffic related to localisation must not use up the entire bandwidth otherwise the applications relying on the WSN data will stop functioning.

Finally, the precision of ranging is another point to be studied. Internal factors as well as external ones will affect performance. We will focus on the effect of multipath, given a PHY layer.

In this paper, we introduce a new protocol for TOF measurement which minimises the power consumption and overhead at the same time. The following section introduces Parallel Double-Sided Two-Way Ranging.

\section{Parallel Double-Sided Two-Way Ranging}

In [9], we examined the results obtained from Sequential SDS-TWR using the CSS technology [2]. While striving to reduce overhead and power consumption, we proposed Parallel Double-Sided TWR (PDS-TWR). The foundation of our study is IEEE 802.15.4. We use the beacon-enabled mode and consider all nodes, anchors and mobiles, as coordinators, thus they all broadcast beacon frames periodically. Collisions between beacons are avoided using the solution introduced in [11]. As the beacon transmission protocol is executed, the mobile becomes aware of the presence of anchors in its neighbourhood. Figure 2(a) illustrates the frame exchanges taking place in PDS-TWR. The mobile broadcasts a LocationStart message containing the list of anchors with which it wishes to perform ranging. The anchors reply one at the time according to their position $p l$ in the list: in this TDMAbased approach, each anchor determines the beginning of its timeslot using $p l$. We thus avoid collisions and the hidden node problem. Once the last Reply1 has been received or a timer in the mobile has expired, the mobile sends the DataRequest: upon reception of this message, the anchors send the collected timestamps to the mobile in the previously defined order. In [10], a theoretical study of the advantages of PDSTWR was presented: by reducing the time spent listening for incoming frames and also sending messages, PDS-TWR reduces the energy consumption of the localisation process. The protocol specification reduces both the number and size of the messages.

Figure 2(b) shows the timestamps collected for a single anchor: the mobile collects $t_{0}, t_{3}$ and $t_{4}$ while the anchor stores $t_{1}, t_{2}$ and $t_{5}$. By specifying the order of the replies, we avoid using a carrier sense scheme and do not need to execute the ranging process again to accommodate anchors which were unable to get a hold of a slot. As a matter of fact, we also defined in [9] the concept of star interval: it is a time slot similar to an IEEE 802.15.4 GTS but allowing communication between a mobile and $n$ selected anchors, forming a star topology. This star interval is created on demand and takes 


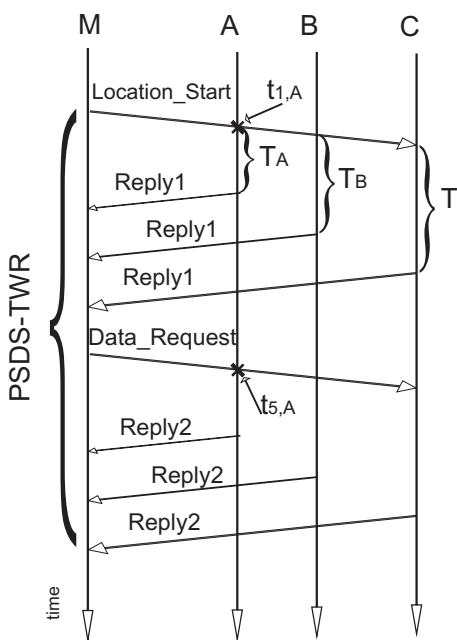

(a) Executing PSDS-TWR with 3 anchors

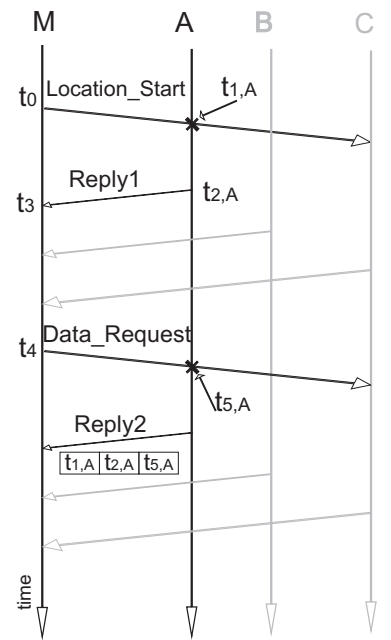

(b) Collecting timestamps for 1 anchor
Fig. 2. Execution of PDS-TWR

TABLE I

COMPARISON OF DIFFERENT RANGING PROTOCOLS IN TERMS OF NUMBER OF FRAMES USED (RANGING ONLY)

\begin{tabular}{|c|c|}
\hline Protocol & Number of frames used for $\mathrm{n}$ anchors \\
\hline TWR & $\mathrm{n} *$ ( Request+ACK) \\
\hline burst & $\mathrm{n}^{*} \mathrm{k}^{*}($ Request+ACK.Request+ACK $)$ \\
\hline D-TWR & $\mathrm{n} *($ 2Request+ACK $)$ \\
\hline SDS-TWR-MA & $\mathrm{n}^{*}($ Request+k*ACK.Request+ACK $)$ \\
\hline PDS-TWR & $2 *$ Request+n*Reply ) \\
\hline
\end{tabular}

place during the sleep period of the network. This way, we avoid collisions with frames from the other nodes.

\section{ANALYTICAL COMPARISON}

In this section, we will compare the following protocols in terms of bandwidth usage and power consumption : TWR, PDS-TWR, SDS-TWR-MA, D-TWR and Burst. SDS-TWRMA can be considered as a general form of SDS-TWR. As AVSR is designed for a network entirely dedicated to localisation, we will not include it in our comparison. We consider that all protocols are executed during a star interval and therefore do not suffer from interference from the same network

\section{A. Bandwidth usage}

Table I provides a comparison of the number of frames used by D-TWR, SDS-TWR-MA and PDS-TWR for $n$ anchors and with $k$ being the number of acknowledgements. We did not include AVSR as its objective is to continuously measure distances to at least three or more anchors. The ranging protocols we are interested in must allow the existence of a communication service and not use the entire available bandwidth.

The numerical application with $\mathrm{k}=2$ and considering an ACK as half of a request frame yields the results of figure 3. When the mobile can reach less than 4 anchors, D-TWR shows the best performance. Otherwise, PDS-TWR has the

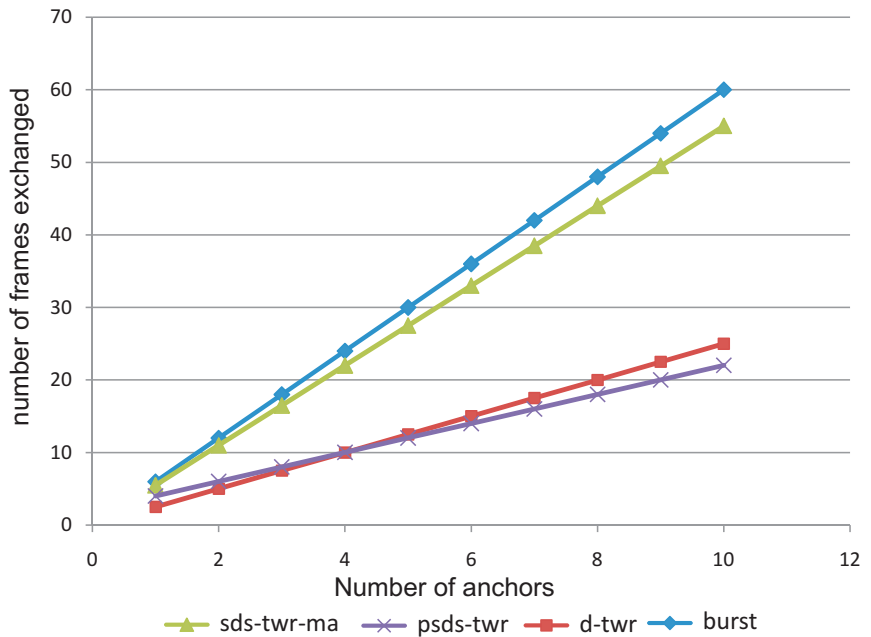

Fig. 3. Comparison of various ranging protocols' medium use

best results. We can conclude that the impact of our protocol on medium use is minimal.

\section{B. Power consumption}

We will now compare our protocols in terms of power consumption. We will first analyse the occupation of the transmit and receive states of the radio for the mobile and anchor roles. In SDS-TWR-MA, the mobile remains in TX while transmitting the Request and ACK frames and is in RX while waiting for the ACK.Request messages. In DTWR, the mobile transmits two messages and switches to RX mode for a single message. In Burst, the mobile transmits $k$ Request messages, listens for $k$ ACK.Request frames and finally transmits $k$ acknowledgements. Finally, in PDS-TWR, the mobile transmits two frames and listens for two messages from each anchor, that is $2 n$ messages. From this description, the consumption for each role can be expressed in terms of duration of the transmissions and power levels associated with each stage, $P_{T X}$ and $P_{R X}$. To enhance readability, we will classify the durations as Td for data transmissions and Ta for acknowledgments. Table II lists the formulae for each role and each protocol.

In Burst, D-TWR and SDS-TWR-MA, the anchors do not know when they will be contacted by the mobile. Therefore, they must remain in RX mode until their turn. Once their participation is over, they may enter sleep mode. As indicated in section III, PDS-TWR assigns a slot to each anchor in a TDMA-based approach. Based on this position, an anchor can further reduce its power consumption by entering sleep mode in the other anchors' slots.

\section{EXPERIMENTAL RESULTS}

Two types of experiments were conducted on our testbed. In the first one, SSDS-TWR was executed using one mobile and three anchors. In the second type of experiment, only two anchors were available. We used them with the mobile to execute PDS-TWR. In both cases, the measurements were 
TABLE II

COMPARISON OF DIFFERENT RANGING PROTOCOLS IN TERMS OF ENERGY CONSUMPTION

\begin{tabular}{|c|c|}
\hline Protocol & Mobile consumption \\
\hline \hline burst & $k \cdot n\left(P_{T X}\left(T_{d}+T_{a}\right)+P_{R X} T_{d}\right)$ \\
\hline D-TWR & $n\left(2 T_{d} \cdot P_{T X}+T_{d} \cdot P_{R X}\right)$ \\
\hline SDS-TWR-MA & $n\left(P_{T X}\left(2 T_{a}+T_{d}\right)+(k+1) \cdot P_{R X} \cdot T_{d}\right)$ \\
\hline PDS-TWR & $2 P_{T X} \cdot T_{d}+2 n P_{R X} \cdot T_{d}$ \\
\hline \hline Protocol & Anchor consumption \\
\hline \hline burst & $n \cdot k\left(P_{T X} T_{d}+P_{R X}\left(T_{d}+T_{a}+\frac{\left(2 T_{d}+T_{a}\right)(n-1)}{2}\right)\right)$ \\
\hline D-TWR & $n\left(2 T_{d} \cdot P_{R X}+T_{d} P_{T X}\right)+3 T_{d} \cdot P_{R X} \cdot \frac{n(n-1)}{2}$ \\
\hline SDS-TWR-MA & $n\left(T_{d} \cdot P_{T X}(k+1)+P_{R X}\left(T_{d}+2 T_{a}\right.\right.$ \\
& $\left.+\frac{(n-1)\left((k+2) T_{d}+2 T_{a}\right)}{2}\right)$ \\
\hline PDS-TWR & $2 . T_{d}\left(P_{T X}+P_{R X}\right)$ \\
\hline
\end{tabular}

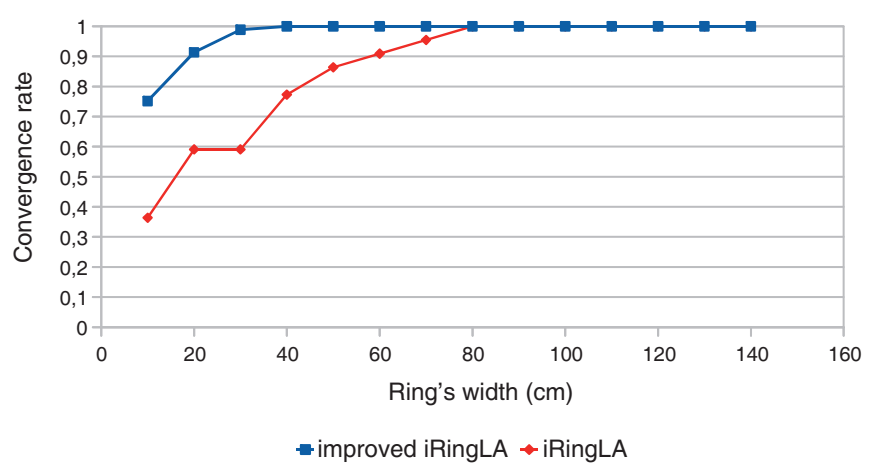

Fig. 4. Impact of the algorithm modification on convergence

processed using the enhanced version of iRingLA. This localisation algorithm computes the intersection of rings centred on the anchors in order to estimate the mobile's position. This version focuses on the most trustworthy search area in order to reduce the possibility of failure. The prototype is built on UWB-based radio transceivers [3], compliant with IEEE 802.15.4-2011, which are controlled by STM32F105RB microcontrollers. The communication took place on the channel centred at $4 \mathrm{GHz}$ (channel 2) with a datarate of $110 \mathrm{kbps}$. The following paragraphs will introduce the results of this prototype-based study.

\section{A. SSDS-TWR data processed by enhanced iRingLA}

Although our algorithm can fit into the memory of most WSN nodes, we decided to implement the same algorithm on a computer in order to study the influence of ring width on the ability to compute the position. We defined convergence as the percentage of cases in which the final position could be computed. Figure 4 shows the impact of iRingLA's modification: the red curve represents the results before and the blue one the results after the enhancement. It becomes clear that this modification improves the chances of finding the position even when thin rings are used.

The impact on mean localisation error was then investigated. The red curves in figure 5 represent the error obtained with the original version of iRingLA and the blue lines illustrate the results obtained with the enhanced version: the curve in the centre represents the average error while the upper and lower

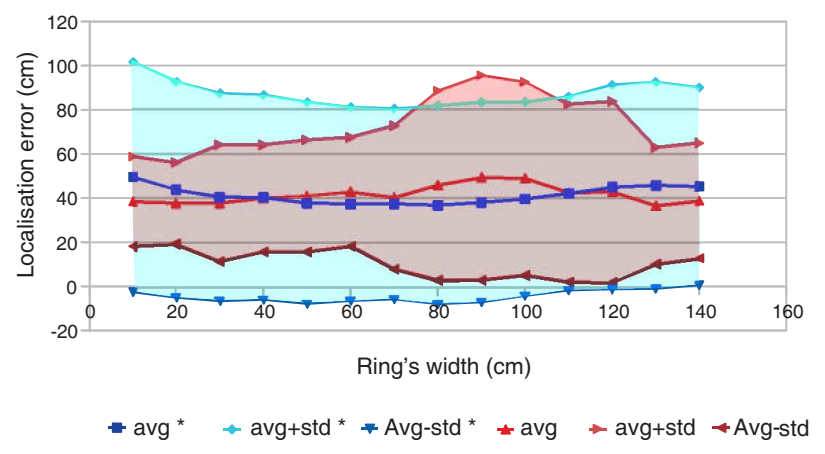

Fig. 5. Impact of the algorithm modification on the mean localisation error

curves correspond to the sum and difference of the average and standard deviation. When the rings are thin (less than $40 \mathrm{~cm}$ ) the error seems to be greater: in fact, when using the enhanced iRingLA, the position can be computed in more cases. Among these are situations where the real position is not within the intersection at all. This results in a large localisation error which shows on figure 5. The apparently lower error obtained with the previous version comes from the fact that we do not take these cases into account. The rest of the blue curve is smoother as the best search area can be identified most of the time: the localisation error remains limited and in the same range as the ranging error.

\section{B. PDS-TWR data processed by enhanced iRingLA}

This experiment involved only 2 anchors and 1 mobile node (figure 6). The anchors were positioned along the $\mathrm{x}$-axis and the area in which the mobile node was placed was considered as the positive values of the y-axis. We then modified the algorithm in order to only select the search area with positive y values. Figure 7 compares the mean localisation error obtained from the same devices when using SSDS-TWR and PDSTWR. Although the topologies are not the same, we will compare the results based on the following observations: in [9], we presented the results obtained using CSS technology in two different rooms, one with metallic furniture (room 1) and the other with bare brick walls (room 2). In room 1, the mean error was slightly greater than in room $2(100.3 \mathrm{~cm}$ instead of $96 \mathrm{~cm})$. Therefore, unless we change the anchors' disposition and create a situation where the Geometric Dilution Of Precision (GDOP) is not in our favour, we can expect similar results from the same topology in the two rooms. UWB is known to be robust to multipath therefore, from a ranging point of view, we can expect the same behaviour regardless of the environment. Since we changed the protocol, we will be able to analyse the impact of this change.

As predicted, the error is a little greater than what we obtained using SSDS-TWR (mean error $79 \mathrm{~cm}$ ). Another cause for the increased error is the reduction of the number of anchors. A third anchor would have caused the selection of a subset of points which is closer to reality. We also observed the need for a correction method even when using UWB. The ranging results obtained using this technology and shown 


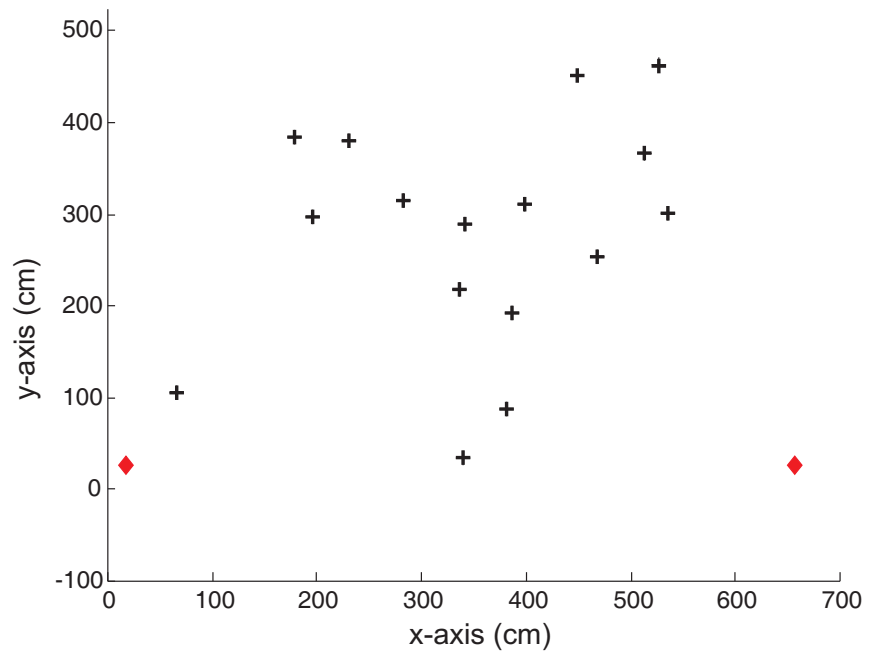

+Mobile position $\quad$ Anchor position

Fig. 6. Topology for PDS-TWR with 2 anchors

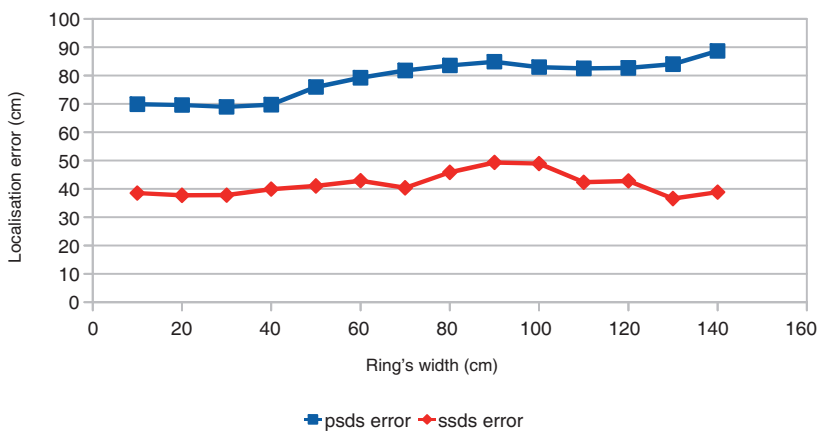

Fig. 7. Impact of the algorithm modification on localisation error

on figure 8 are precise but not accurate. In this experiment, thirty samples were collected for each measurement point. The distance estimates have a very small dispersion most of the time but the ranging error remains significant. A range correction method is therefore necessary to move the results closer to the real value. We are currently investigating the correlation between the protocol and the increased error in order to propose a scalable correction method.

\section{CONCLUSION}

In our pursuit of autonomous indoor localisation, we have chosen to build a solution on TOF as it is a reliable source of information. We proposed a new protocol for distance measurement and also an efficient algorithm which can be executed by any node, no matter how constrained its resources. We implemented our solution on a UWB-based prototype and presented the results of localisation in this article. The enhanced iRingLA focuses on the most trustworthy search

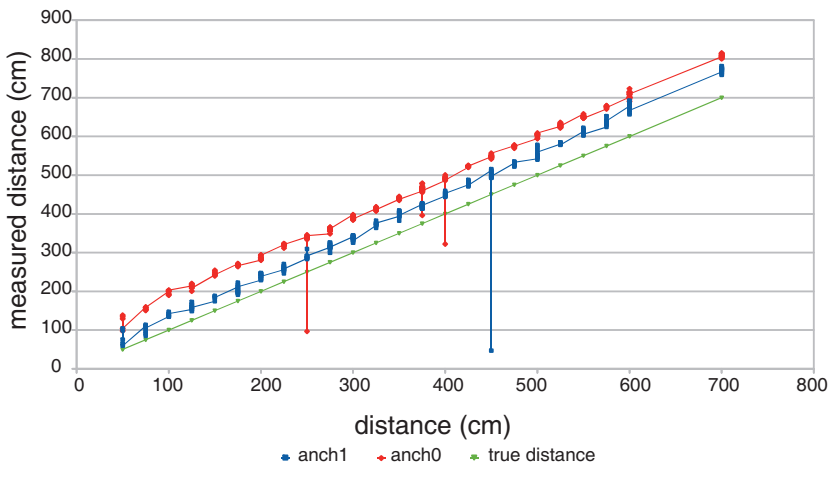

Fig. 8. Ranging error using PDS-TWR and 2 anchors

area in order to reduce the possibility of failure. We noticed that the convergence rate improved and the error, for data collected using SSDS-TWR, was close to the ranging error. We also compared PDS-TWR to other existing ranging protocols: PDS-TWR uses the smallest number of messages, thus making the ranging process faster. A direct and positive impact on energy consumption can be expected, making PDS-TWR a green protocol. The expected loss of accuracy is limited and therefore, PDS-TWR can be used with iRingLA in indoor localisation scenarios. Our study so far has focused on LOS scenarios, although some of our experiments took place in crowded rooms. In the future, we plan on studying the impact of NLOS on our solution. We also consider designing a more efficient correction method for the ranging results and also conducting a prototype-based study of the integration of our solution in the time structure of an IEEE 802.15.4 network.

\section{REFERENCES}

[1] IEEE Computer Society, Specific requirements Part 15.4: Wireless Medium Access Control (MAC) and Physical Layer (PHY) Specifications for Low-Rate Wireless Personal Area Networks (WPANs) Amendment 1: Add Alternate PHYs, IEEE Standard for Information technology Telecommunications and information exchange between systems (2007)

[2] Nanotron http://www.nanotron.com/EN/pdf/Factsheet_nanoLOC-AVRModule.pdf (Accessed december 2011)

[3] Decawave http://www.decawave.com/products/dwm1000-module (Accessed February 2014)

[4] A.I. Baba, M.M. Atia, Burst mode symmetric double sided two way ranging, IFIP Wireless Days (WD) (2011)

[5] M. Kwak, J. Chong, A new Double Two-Way Ranging algorithm for ranging system, 2nd IEEE International Conference on Network Infrastructure and Digital Content (2010)

[6] H. Kim, Double-Sided Two-Way Ranging Algorithm to Reduce Ranging Time, IEEE Communication Letters, Vol. 13, no. 7, July 2009

[7] H. Kim, Performance Comparison of Asynchronous Ranging Algorithms, IEEE GLOBECOM (2009)

[8] K. Hwang, AVSR: Asynchronous Virtual Slot-based Ranging for USerOriented Location Services, IEEE Transactions on Consumer Electronics (2011)

[9] R. Dalce, A. van den Bossche, T. Val, Indoor Self-Localization in a WSN, based on Time Of Flight: Propositions and Demonstrator, International Conference on Indoor Positionning and Indoor Navigation (2013)

[10] R. Dalce, Localization methods using the communication signal in indoor wireless sensor networks. Phd thesis, INSA Toulouse - EDSYS - Laboratories LATTIS and IRIT (2013)

[11] J. Lu, A. van den Bossche, E. Campo, Enabling Guaranteed Beacon and Data Slots in Multi-hop Mesh Sensor Networks for Home Health Monitoring International Conference on Wireless and Mobile Communications (2012) 